\title{
Divisions asymétriques ovocytaires
}

(CIRB)

\section{Marie-Hélène Verlhac}

\section{OpenEdition Journals}

Édition électronique

URL : https://journals.openedition.org/annuaire-cdf/1092

DOI : 10.4000/annuaire-cdf.1092

ISBN : 978-2-7226-0325-7

ISSN : 2109-9227

Éditeur

Collège de France

\section{Édition imprimée}

Date de publication : 1 avril 2013

Pagination : 899-902

ISBN : 978-2-7226-0198-7

ISSN : 0069-5580

Référence électronique

Marie-Hélène Verlhac, " Divisions asymétriques ovocytaires », L'annuaire du Collège de France [En ligne], 112 | 2013, mis en ligne le 22 novembre 2013, consulté le 22 août 2022. URL : http://

journals.openedition.org/annuaire-cdf/1092; DOI : https://doi.org/10.4000/annuaire-cdf.1092 


\title{
PUBLICATIONS
}

Heller E.A., Zhang W., Selimi F., Earnheart J.C., Slimak M.A., Santos-Torres J., IbañezTallon I., Aoki C., Chait B.T. \& Heintz N., « The biochemical anatomy of cortical inhibitory synapses », PLoS One, 7(6), 2012, e39572.

Selimi F., Cristea I.M., Heller E., Chait B.T. \& Heintz N., « Proteomic studies of a single CNS synapse type: specific regulatory components of the parallel fiber/Purkinje cell synapse », PloS Biol., 7(4), 2009, e83.

\section{Divisions asymétriques ovocytaires (CIRB)}

\author{
Responsable : Marie-Hélène VERLHAC
}

\section{RECHERCHE}

Les ovocytes de métazoaires subissent deux divisions successives, asymétriques par la taille des cellules filles engendrées, donnant naissance à une très grosse cellule germinale, l'ovocyte et deux petits globules polaires. Ceci assure le maintien des réserves maternelles, indispensable au développement embryonnaire. L'asymétrie des divisions méiotiques de l'ovocyte de souris repose sur la formation d'un fuseau de division en absence de centrosomes canoniques et son positionnement au cortex de la cellule, qui dépend principalement des microfilaments d'actine (Dumont, 2007a ; Azoury 2008). Comment s'assemblent les fuseaux méiotiques en absence de centrosomes canoniques? Comment les forces de tension aux kinétochores des chromosomes sont-elles transmises en l'absence de pôles robustes ancrés dans le cortex cellulaire? Quelles sont les modalités de mise en place des différents réseaux d'actine contrôlant le positionnement?

\section{1) Le fort taux d'erreurs en méiose I lié à une faiblesse du point de contrôle du fuseau !}

En mitose, les fuseaux de division s'organisent par la voie des centrosomes et la voie des chromosomes. En méiose, la voie des chromosomes est importante mais, d'une manière inattendue, elle n'implique pas le gradient de RanGTP (Dumont, 2007b ; Brunet, 2008). Les erreurs d'organisation des fuseaux méiotiques induisent des aneuploïdies à l'origine de maladies génétiques, de nombreux cancers, voire totalement incompatibles avec le développement embryonnaire. La méiose I chez l'homme est réputée pour avoir un taux d'erreur de ségrégation des chromosomes très élevée, sans que l'on en connaisse les raisons. Ce fort taux d'aneuploïdies est à l'origine de nombreuses trisomies, notamment chez les femmes âgées de plus de 35 ans. La formation ainsi que les mécanismes de positionnement originaux des fuseaux acentriolaires méiotiques soulèvent la question fondamentale de la transmission 
des forces de tension au sein du fuseau méiotique, en l'absence de pôles de fuseaux robustes ancrés dans des centrosomes, et de connexion entre centrosomes et cortex via les microtubules. Afin de tester l'ancrage des MTOCs aux microtubules, nous avons étudié un mutant conditionnel de NumA (Nuclear Mitotic Apparatus), une MAP essentielle à l'ancrage des pôles des fuseaux mitotiques aux centrosomes. $\mathrm{Ce}$ travail sur l'allèle mutant de NumA ne s'associant plus aux microtubules a été réalisé en collaboration avec le laboratoire de Don Cleveland (États-Unis). L'expression du mutant a lieu au cours de l'ovogenèse grâce à l'utilisation de souris exprimant la Cre-recombinase sous contrôle du promoteur ZP3 (Zona Pellucida 3, spécifique de la lignée germinale femelle). Les souris femelles exprimant cet allèle sont stériles, présentant des défauts d'organisation des fuseaux méiotiques conduisant à la formation d'œufs aneuploïdes. Nos travaux ont démontré que le Spindle Assembly Checkpoint (SAC) ne détecte pas la diminution des forces de tension inter-kinétochores qui touche les chromosomes de ces fuseaux mutants. Ce résultat très important (Kolano, PNAS, 2012) a été aussi suggéré mais non démontré, comme dans notre étude, par des approches différentes (Gui \& Homer, Dev., 2012 ; Lane, Dev., 2012). Il suggère que les erreurs de ségrégation en méiose I sont liées à une faible sensibilité du SAC méiotique à la réduction des forces de tension au niveau des kinétochores.

\section{2) Contrôle de la division asymétrique ovocytaire}

\section{Remodelage de l'actin cytoplasmique}

De par l'absence de centrosomes, la plupart des fuseaux méiotiques sont dépourvus d'asters microtubulaires les connectant à la membrane plasmique cellulaire. Le positionnement de ces fuseaux au cortex ovocytaire ne dépend donc pas de microtubules astèriens, mais dépend de microfilaments d'actine. Or, leur positionnement au cortex dans des cellules de taille gigantesque, par rapport aux cellules somatiques, est indispensable aux divisions asymétriques permettant l'expulsion de petits globules polaires. Nous avons montré que la Formine-2, un nucléateur de l'actine, est essentielle à la formation d'un réseau de microfilaments d'actine cytoplasmique s'assemblant autour du fuseau méiotique et assurant la migration et l'ancrage de ce dernier au cortex (Azoury, 2008). Nous avons étudié le remodelage in vivo de ce réseau au cours du temps et pu observer que ce réseau disparaissait avant l'entrée en méiose I puis réapparaissait en cours de méiose I en corrélation avec la présence de la Formine 2. La surexpression de la Formine-2 permet de saturer le système de dégradation normalement présent à la reprise de la méiose, empêche la déstabilisation du réseau de microfilaments d'actine, et a pour conséquence de bloquer la migration du fuseau de méiose I au cortex. Ce remodelage de l'actine cytoplasmique est donc essentiel à la division asymétrique (Azoury, 2011).

\section{La baisse de rigidité corticale, associée à un enrichissement en microfilaments mais un appauvrissement en myosine II, permet la migration du fuseau au cortex cellulaire}

Lors de leur entrée en mitose, les cellules s'arrondissent et voient leur rigidité corticale augmenter, ce qui permet une transmission efficace des forces de traction issues de l'environnement afin d'assurer correctement l'assemblage et l'orientation du fuseau de division. Les fuseaux méiotiques d'ovocytes de souris n'ont pas de centrosomes 
ni de microtubules astraux. A l'inverse de la mitose, la tension corticale chute en phase $\mathrm{M}$ dans les ovocytes, sans qu'on sache les raisons moléculaires qui sous-tendent cette diminution (Larson, 2010). Nous avons mis en évidence un épaississement de l'actine corticale non encore documenté. Cet épaississement est progressif et est essentiel à la migration du fuseau au cortex. Il est nucléé par Arp2/3 et régulé par la voie Mos.../MAPK, via la phosphorylation/activation de Wave 2. Cet épaississement est associé à une diminution de la quantité de myosine II au cortex cellulaire. Des expériences de photoactivation montrent que la dynamique des filaments d'actine dans le cytoplasme et dans le cortex est très différente et contribue à l'établissement de deux compartiments d'actine distincts. En utilisant la technique d'aspiration avec des micropipettes (collaboration avec l'équipe de Cécile Sykes, Institut Curie), nous avons montré que l'épaississement d'actine diminue la tension corticale et confère une plasticité à l'ovocyte, propriété importante afin de faciliter l'expulsion d'un petit globule polaire à l'anaphase. Au contraire, l'actine dans le cytoplasme confère une tension corticale élevée et de l'élasticité à l'ovocyte. La diminution de la tension corticale au cours de la première division méiotique est essentielle pour son asymétrie puisque le maintien artificiel d'une tension corticale élevée empêche la migration du fuseau au cortex. En conclusion, la migration du fuseau dans les ovocytes de souris est finement régulée par une chute de tension corticale qui dépend d'un épaississement de l'actine au cortex (Chaigne, soumis pour publication).

\section{Conclusions}

Nos travaux récents suggèrent que les ovocytes ont des propriétés communes avec certaines cellules cancéreuses : tri des centrosomes surnuméraires dans certaines tumeurs solides/ tri des MTOCs acentriolaires aux pôles des fuseaux méiotiques (Breuer, 2010) ; perte d'adhérence des cellules cancéreuses/perte d'adhérence en cours de division méiotique assurée par un épaississement du cortex. Il sera intéressant de poursuivre ce parallèle afin de déterminer si nos observations du comportement du cortex dans les ovocytes se retrouvent aussi dans certains types de cancer. Enfin, la découverte récente d'un lien entre régulation des divisions asymétriques et cancer, suggère que comprendre les modes de positionnement du fuseau dans ce type de divisions s'avère un enjeu important en cancérologie. Là encore, il apparaît très important d'élucider les mécanismes fondamentaux de positionnement du fuseau dépendants de l'actine et fortement présents dans notre modèle de division asymétrique ovocytaire.

\section{Références}

Azoury J., Lee K.W., Georget V., Rassinier P., Leader B. et Verlhac M.-H., «Spindle positioning in mouse oocytes relies on a dynamic meshwork of actin filaments », Curr. Biol., $18,2008,1514-19$.

Azoury J., Lee K.W., Georget V. Hikal P. et Verlhac M.-H., « Symmetry breaking in mouse oocytes requires transient F-actin meshwork destabilization », Development, 138, 2011, 2903-8.

Breuer M., Kolano A., Kwon M., Li C.-C., Tsai T.-F., Pellman D., Brunet S. et Verlhac M.-H., «HURP permits MTOC sorting for robust meiotic spindle bipolarity, similar to extra-centrosome-clustering in cancer cells », J. Cell. Biol., 19, 2010, 1251-1260. 
Brunet S., Dumont J., Lee K.W., Kinoshita K., Hikal P., Gruss O., Maro B. et Verlhac M.-H., « Meiotic regulation of TPX2 protein levels governs cell cycle progression in mouse oocytes », PLos One, 3, 2008, e3338.

Chaigne A., Campillo C., Almonacid M., Azoury J., Perez F., Nassoy P., Sykes C., Verlhac M.-H.* et Terret M.-E.*, «Cortical stiffness prevents spindle positioning in mouse oocytes », soumis pour publication.

Dumont J., Million K., Sunderland K., Rassinier P., Lim H., Leader B. et Verlhac M.-H., «Formin-2 is required for spindle migration and for the late steps of cytokinesis in mouse oocytes », Dev. Biol., 301, 2007a, 254-65.

Dumont J., Petri S., Pellegrin F., Terret M.-E., Bohnsack M.T., Rassinier P., Georget V., Kalab P., Gruss O.J. et Verlhac M.-H., «A centriole and RanGTP independent spindle assembly pathway in meiosis I of vertebrate oocytes », J. Cell. Biol., 176, 2007b, 295-305.

Gui L., Homer H., « Spindle assembly checkpoint signalling is uncoupled from chromosomal position in mouse oocytes », Development, 139, 2012, 1941-6.

Lane S.I., Yun Y., Jones K.T. 2012. «Timing of anaphase-promoting complex activation in mouse oocytes is predicted by microtubule-kinetochore attachment but not by bivalent alignment or tension », Development, 139,1947-55.

Larson S.M. et al., «Cortical mechanics and meiosis II completion in mammalian oocytes are mediated by myosin-II and Ezrin-Radixin-Moesin (ERM) proteins », Mol. Biol. Cell., 21, 2010, 3182-3192.

Kolano A., Brunet S., Silk A.D., Cleveland D.W. et Verlhac M.-H., « Error prone mammalian female meiosis from silencing the SAC without normal interkinetochore tension », PNAS, 109, 2012, E1858-E1867.

\section{Publications 2011-2012}

Kolano A., Brunet S., Silk A.D., Cleveland D.W. et Verlhac M.-H., «Error prone mammalian female meiosis from silencing the SAC without normal interkinetochore tension », PNAS, 109, 2012, E1858-E1867.

Chaigne A., Verlhac M.-H. et Terret M.E., « Spindle positioning in mammalian oocytes », Exp. Cell Res., 318, 2012, 1442-1447.

Dumont J. et Verlhac M.-H., «Using FRET to study RanGTP gradients in live mouse oocytes », in Hayden H. (éd.), Methods in Molecular Biology, Springer, 957, 2012, 107-120.

Verlhac M.-H. et Breuer M., «Cytoskeletal correlates of oocyte meiotic divisions », in Pierce M., Coticchio G. et Albertini D. (éd.), Mammalian, Springer, chapter 14, 2012, 195-207.

Verlhac M.-H., « Meeting report of the minisymposium on "Meiosis and Oogenesis" », Mol. Biol. Cell, 23, 2012, 971-971.

Verlhac M.-H., «Spindle positioning: going against the actin flow », Nat. Cell Biol., 12, 2011, 1183-1185.

Azoury J., Lee K.W., Georget V., Hikal P. et Verlhac M.-H., « Symmetry breaking in mouse oocytes requires transient F-actin meshwork destabilization », Development, 138, 2011, 2903-2908.

Brunet S. et Verlhac M.-H., «Positioning to get out of Meiosis: the asymmetry of division », Hum. Reprod. Update, 17, 2011, 68-75. 